\title{
STOCHASTIC STABILITY IN SPATIAL GAMES
}

\author{
JACEK MIĘKISZ \\ Institute of Applied Mathematics and Mechanics, University of Warsaw \\ Banacha 2, 02-097 Warsaw, Poland \\ E-mail:miekisz@mimuw.edu.pl
}

\begin{abstract}
We compare two concepts of stochastic stability in spatial games. The classical approach to stochastic stability, introduced by Foster and Young [8], involves single configurations in the zero-noise limit. Ensemble stability discussed in [17] refers to ensembles of configurations in the limit of an infinite number of players. The above two limits may not commute. We will discuss reasons of such behaviour. We review some results concerning the effect of the number of players and the noise level on the long-run behaviour of stochastic dynamics of spatial games.
\end{abstract}

1. Introduction. Many evolutionary and ecological processes can be modeled as systems of interacting players $[16,33,32,27,12,35,5,13,24,25]$. In such models, players have at their disposal certain strategies and their payoffs depend on strategies chosen both by them and by their opponents. The central concept in game theory is that of a Nash equilibrium. It is an assignment of strategies to players such that no player, for fixed strategies of his opponents, has an incentive to deviate from his current strategy; no change can increase his payoff. One of the fundamental problems in game theory is the equilibrium selection in games with multiple Nash equilibria. One of the selection methods is to construct an appropriate dynamical system where in the long run only one equilibrium is played with a high frequency.

Here we will discuss spatial games with certain local interactions. In such games, players are located on vertices of certain graphs and they interact only with their neighbours; see for example $[21,22,23,1,6,35,7,15,30,11]$ and a recent review [31] and references therein. In discrete moments of time, players adapt to their neighbors by choosing with a high probability the strategy which is the best response, i.e. the one which maximizes

2000 Mathematics Subject Classification: 91A10, 91A22.

Key words and phrases: evolutionary game theory, Nash equilibria, spatial games, equilibrium selection, stochastic stability.

I would like to thank the Ministry of Science and Higher Education for a financial support under the grant N201 023 31/2069.

The paper is in final form and no version of it will be published elsewhere. 
the sum of the payoffs obtained in individual games and with a small probability they make mistakes.

To describe the long-run behavior of such stochastic dynamics, Foster and Young [8] introduced a concept of stochastic stability. A configuration of a system - an assignment of stategies to players - is stochastically stable if it has a positive probability in the stationary distribution of the above dynamics in the limit of zero noise. It means that in the long run we observe it with a positive frequency. In classical applications of stochastic stability for equilibrium selection $[14,26,32,34,35]$, the population size was fixed and the noise level was taken to zero. However, we may also consider models with a fixed noise level in the limit of an infinite number of players [27, 17, 18, 19, 20, 28]. Now, for any arbitrarily low but fixed noise, if the number of players is big enough, then the probability of any individual configuration is practically zero. It means that for a big number of players, to observe a stochastically stable configuration we must assume that players make mistakes with extremely small probabilities. On the other hand, it may happen that in the long run, for a low but fixed noise and sufficiently big number of players, the stationary distribution is highly concentrated on an ensemble consisting of one Nash configuration and its small perturbations, i.e. configurations where most players play the same strategy. We will call such configurations ensemble stable [17].

In Section 2, we formally introduce spatial games and two concepts of stochastic stability. In Section 3, we discuss some other results and state an open problem.

2. Spatial games and stochastic stability. Let $\Lambda$ be a finite subset of the simple lattice $\mathbf{Z}^{d}$ (we may think about a square centered at the origin of the lattice). Every site of $\Lambda$ is occupied by one player who has at his disposal one of $k$ different pure strategies. Let $S$ be a set of pure strategies, then $\Omega_{\Lambda}=S^{\Lambda}$ is the set of all possible configurations of players, that is all possible assignments of pure strategies to individual players. For every $i \in \Lambda, X_{i}$ is the strategy of the $i$-th player in the configuration $X \in \Omega_{\Lambda}$ and $X_{-i}$ denotes strategies of all remaining players; $X$ therefore can be represented as the pair $\left(X_{i}, X_{-i}\right)$. $U: S \times S \rightarrow R$ is a matrix of payoffs of our stage game. $U(A, B), A, B \in S$ is the payoff of the first (row) player playing the strategy $A$ when the second one (a column player) is playing $B$. We will consider here only symmetric games so the payoff of the second player is given by $U(B, A)$ (the payoff matrix of the second player is the transpose of the payoff matrix $U$ of the first one). Every player interacts only with his neighbours and his payoff is the sum of the payoffs resulting from individual games. We assume that he has to use the same strategy for all neighbours. Let $N_{i}$ denote the neighbourhood of the $i$-th player. For the nearest-neighbor interaction we have $N_{i}=\{j ;|j-i|=1\}$, where $|i-j|$ is the distance between $i$ and $j$. For $X \in \Omega_{\Lambda}$ we denote by $\nu_{i}(X)$ the payoff of the $i$-th player in the configuration $X$ :

$$
\nu_{i}(X)=\sum_{j \in N_{i}} U\left(X_{i}, X_{j}\right)
$$

Definition 1. $X \in \Omega_{\Lambda}$ is a Nash configuration if for every $i \in \Lambda$ and $A \in S$

$$
\nu_{i}\left(X_{i}, X_{-i}\right) \geq \nu_{i}\left(A, X_{-i}\right) .
$$


We describe now the deterministic dynamics of the best-response rule. Namely, at each discrete moment of time $t=1,2, \ldots$, a randomly chosen player may update his strategy. He simply adopts the strategy, $X_{i}^{t}$, which gives him the maximal total payoff $\nu_{i}\left(X_{i}^{t}, X_{-i}^{t-1}\right)$ for given $X_{-i}^{t-1}$, a configuration of strategies of remaining players at time $t-1$.

Now we allow players to make mistakes with a small probability, that is to say they may not choose the best response. A probability of making a mistake may depend on the state of the system (a configuration of strategies of neighboring players). We will assume that this probability is a decreasing function of the payoff lost as a result of a mistake $[1,2]$. In the $\log$-linear rule, the probability of chosing by the $i$-th player the strategy $X_{i}^{t}$ at time $t$ is given by the following conditional probability:

$$
p_{i}^{\epsilon}\left(X_{i}^{t} \mid X_{-i}^{t-1}\right)=\frac{e^{(1 / \epsilon) \nu_{i}\left(X_{i}^{t}, X_{-i}^{t-1}\right)}}{\sum_{A \in S} e^{(1 / \epsilon) \nu_{i}\left(A, X_{-i}^{t-1}\right)}},
$$

where $\epsilon>0$ measures the noise level.

Let us observe that if $\epsilon \rightarrow 0, p_{i}^{\epsilon}$ converges to the best-response rule. Our stochastic dynamics is an example of an ergodic Markov chain with $\left|S^{\Lambda}\right|$ states. Therefore, it has a unique stationary distribution which we denote by $\mu_{\Lambda}^{\epsilon}$.

The following definition was first introduced by Foster and Young [8]:

Definition 2. $X \in \Omega_{\Lambda}$ is stochastically stable if $\lim _{\epsilon \rightarrow 0} \mu_{\Lambda}^{\epsilon}(X)>0$.

If $X$ is stochastically stable, then the frequency of visiting $X$ converges to a positive number along any time trajectory almost surely. It means that in the long run we observe $X$ with a positive frequency. In most models it is usually equal to 1 . In examples below, we consider symmetric games with symmetric Nash equilibria and homogeneous Nash configurations. By stochastic stability of a strategy or a Nash equilibrium we mean stochastic stability of the corresponding Nash configuration.

EXAmple 1. Players are located on a finite subset $\Lambda$ of $\mathbf{Z}$ (with periodic boundary conditions) and interact with their two nearest neighbors. They have at their disposal two strategies: $A$ and $B$. The payoffs are given by the following symmetric matrix:

$$
U=\begin{array}{lll} 
& A & B \\
A & 5 & 0 \\
B & 3 & 3
\end{array}
$$

It is easy to see that the above stage game has two Nash equilibria: $(A, A)$ and $(B, B)$, and the corresponding spatial game has two homogeneous Nash configurations: $X^{A}$ and $X^{B}$, in which all individuals play the same strategy, $A$ or $B$ respectively.

One can show that $X^{B}$ is stochastically stable $[1,34]$. The reason for such a long-run behavior is that the population needs many mistakes (actually the number of required mistakes is proportional to the population size) to move from $X^{B}$ to $X^{A}$. On the contrary, one needs only one mistake to move from $X^{A}$ to $X^{B}$; one $A$-player makes a mistake and switches to $B$ and then both his neighbours may switch to $B$ by the noise-free dynamics of the best-response rule. We see that no more mistakes are required for $B$ strategy to invade the whole population. One can make this argument rigorous using a tree representation of stationary distributions of ergodic Markov chains $[9,14,34,26,32,35,6,7,20]$. 
In general, we have to investigate all absorbing states of the noise-free dynamics. When we start with any non-absorbing state, then after a finite number of steps of the noise-free best-response dynamics we arrive at one of the absorbing states and then stay there forever. It follows from the tree representation of the stationary distribution that any state different from absorbing states has zero probability in the stationary distribution in the zero-noise limit.

Stochastically stable states are those absorbing states from which it is relatively more difficult to get out than from other absorbing states $[6,7]$.

The situation gets more complex when for a fixed noise level we are approaching the infinite population limit. It may happen then that in the long run one sees configurations from which it is relatively easier for a player to switch to a different strategy than from other absorbing configurations. This is the case in the following example.

EXAmple 2. Players are located on a finite subset $\Lambda$ of $\mathbf{Z}^{2}$ (with periodic boundary conditions) and interact with their four nearest neighbours. They have at their disposal three pure strategies: $A, B$, and $C$. The payoffs are given by the following symmetric matrix:

$$
U=\begin{array}{cccc} 
& A & B & C \\
A & 2 & 0 & 1 \\
B & 0 & 3+\alpha & 0 \\
C & 1 & 0 & 3
\end{array}
$$

where $\alpha \geq 0$.

Our game has three Nash equilibria: $(A, A),(B, B)$, and $(C, C)$, and the corresponding spatial game has three homogeneous Nash configurations: $X^{A}, X^{B}$, and $X^{C}$.

The unique stationary distribution of the log-linear dynamics of the above game can be explicitly constructed [35] and is given by

$$
\mu_{\Lambda}^{\epsilon}(X)=\frac{e^{(1 / \epsilon) \sum_{(i, j) \in \Lambda} U\left(X_{i}, X_{j}\right)}}{\sum_{Z \in \Omega_{\Lambda}} e^{(1 / \epsilon) \sum_{(i, j) \in \Lambda} U\left(Z_{i}, Z_{j}\right)}} .
$$

Let us start our discussion with the case of $\alpha=0$. It follows from (3) that

$$
\lim _{\epsilon \rightarrow 0} \mu_{\Lambda}^{\epsilon}\left(X^{k}\right)=1 / 2,
$$

for $k=B, C$ so $B$ and $C$ are stochastically stable.

Let us investigate the long-run behavior of our system for large $\Lambda$, that is for a big number of players. Observe that

$$
\lim _{\Lambda \rightarrow \mathbf{Z}^{2}} \mu_{\Lambda}^{\epsilon}(X)=0
$$

for every $X \in \Omega=S^{\mathbf{Z}^{2}}$.

Hence for large $\Lambda$ and $\epsilon>0$ we may only observe, with reasonable positive frequencies, ensembles of configurations and not particular configurations. We will be interested in ensembles which consist of a Nash configuration and its small perturbations, that is configurations, where most players adopt the same strategy. 
We perform first the limit $\Lambda \rightarrow \mathbf{Z}^{2}$ and obtain an infinite-population state, the so called Gibbs state [10, 29],

$$
\mu^{\epsilon}=\lim _{\Lambda \rightarrow \mathbf{Z}^{2}} \mu_{\Lambda}^{\epsilon} .
$$

We use techniques of statistical mechanics and the concept of dominant ground states $[3,4]$, modify proofs in [17] and get

$$
\mu^{\epsilon}\left(X_{i} \neq C\right)<\gamma(\epsilon)
$$

for any $i \in \mathbf{Z}^{2}$ and $\gamma(\epsilon) \rightarrow 0$ as $\epsilon \rightarrow 0$.

Let us sketch briefly the reason of such a result. While it is true that both $X^{B}$ and $X^{C}$ have the same maximal total payoff (and therefore are stochastically stable), the $X^{C}$ Nash configuration has lower-cost excitations. Namely, one player can change its strategy and switch to $A$ and the total payoff will decrease by 16 units. Players in the $X^{B}$ Nash configuration can switch either to $A$ or $B$ to decrease the total payoff by 24 . Now, the probability of the occurrence of any configuration in the Gibbs state (which is the stationary distribution of our stochastic dynamics) depends on the total payoff in an exponential way. One can prove that the probability of the ensemble consisting of the $X^{C}$ Nash configuration and configurations which are different from it at few sites only is much bigger than the probability of the analogous $X^{B}$-ensemble. On the other hand, configurations which are outside $X^{B}$ and $X^{C}$-ensembles appear with exponentially small probabilities. It means that for large enough systems (and small but not extremely small $\epsilon)$ we observe in the stationary distribution the $X^{C}$ Nash configuration with perhaps few different strategies. The above argument was made into a rigorous proof for an infinite system of the closely related lattice-gas model (the Blume-Capel model) of interacting particles by Bricmont and Slawny in [3].

We see that for any low but fixed $\epsilon$, if the number of players is big enough, then in the long run, almost all players use $C$ strategy. On the other hand, if for any fixed number of players, $\epsilon$ is lowered substantially, then $B$ and $C$ appear with frequencies close to $1 / 2$.

The above example motivates following definitions [17].

Definition 3. $X \in \Omega_{\Lambda}$ is $\gamma$-ensemble stable if $\mu_{\Lambda}^{\epsilon}\left(Y \in \Omega_{\Lambda} ; Y_{i} \neq X_{i}\right)<\gamma$ for any $i \in \Lambda$ if $\Lambda \supset \Lambda(\gamma)$ for some $\Lambda(\gamma)$.

Definition 4. $X \in \Omega_{\Lambda}$ is low-noise ensemble stable if for every $\gamma>0$ there exists $\epsilon(\gamma)$ such that if $\epsilon<\epsilon(\gamma)$, then $X$ is $\gamma$-ensemble stable.

The following theorem is a simple consequence of (5).

THEOREM 1. If $\alpha=0$, then $X^{C}$ is low-noise ensemble stable.

If $X$ is $\gamma$-ensemble stable with $\gamma$ close to zero, then the ensemble consisting of $X$ and configurations which are different from $X$ at at most few sites has the probability close to one in the stationary distribution. It does not follow, however, that $X$ is necessarily low-noise ensemble or stochastically stable as it happens in our example in the case of $\alpha>0$.

For $\alpha=0$, both $X^{B}$ and $X^{C}$ have the same total payoff. $X^{C}$ has lower-cost fluctuations and therefore it is low-noise ensemble stable. For $\alpha>0, X^{C}$ has a smaller 
total payoff but nevertheless one can prove [17] that in the long run $C$ is played with a frequency close to 1 if the noise level is low but not extremely low.

TheORem 2. For every $\gamma>0$, there exist $\alpha(\gamma)$ and $\epsilon(\gamma)$ such that for every $0<\alpha<$ $\alpha(\gamma)$, there exists $\epsilon(\alpha)$ such that for $\epsilon(\alpha)<\epsilon<\epsilon(\gamma), X^{C}$ is $\gamma$-ensemble stable, and for $0<\epsilon<\epsilon(\alpha), X^{B}$ is $\gamma$-ensemble stable.

Observe that for $\alpha=0$, both $X^{B}$ and $X^{C}$ are stochastically stable (they appear with the frequency $1 / 2$ in the limit of zero noise) but $X^{C}$ is low-noise ensemble stable. For small $\alpha>0, X^{B}$ is both stochastically (it appears with the frequency 1 in the limit of zero noise) and low-noise ensemble stable. However, for an intermediate noise $\epsilon(\alpha)<\epsilon<\epsilon(\gamma)$, if the number of players is large enough, then in the long run, almost all players use the strategy $C\left(X^{C}\right.$ is ensemble stable). If we lower $\epsilon$ below $\epsilon(\alpha)$, then almost all players start to use the strategy $B . \epsilon=\epsilon(\alpha)$ is the line of the first-order phase transition. In the infinite population limit there exist two stationary distributions on this line. We may say that at $\epsilon=\epsilon(\alpha)$, the population of players undergoes a sharp equilibrium transition from $C$ to $B$-behaviour.

3. Discussion. We have discussed two concepts of stochastic stability in spatial games. Classical stochastic stability involves single configurations in the limit of zero noise, ensemble stability refers to ensembles of configurations in the infinite population limit. We have shown that the above two limits may not commute. Therefore, to study the long-run behavior of real populations, we should estimate the relevant parameters to be sure what limiting procedures are appropriate in specific examples.

Similar ideas were recently discussed in two-player games in well-mixed populations [28]. States of such systems are described by strategy frequencies. One takes first the limit of an infinite number of players. Stationary distributions are then measures concentrated on single frequencies. It was shown in [28] that a stationary frequency may change discontinously at a certain noise level.

Let us end our paper by presenting an open problem.

Open Problem. Construct a stochastic evolutionary spatial game with a unique stationary distribution $\mu_{\Lambda}^{\epsilon}$ such that

$$
\begin{aligned}
& \lim _{\epsilon \rightarrow 0} \lim _{\Lambda \rightarrow \mathbf{Z}^{2}} \mu_{\Lambda}^{\epsilon}(X)=1, \\
& \lim _{\Lambda \rightarrow \mathbf{Z}^{2}} \lim _{\epsilon \rightarrow 0} \mu_{\Lambda}^{\epsilon}(Y)=1,
\end{aligned}
$$

where $X, Y \in \Omega=Z^{d}, X \neq Y$.

\section{References}

[1] L. E. Blume, The statistical mechanics of strategic interaction, Games Econ. Behav. 5 (1993), 387-424.

[2] L. E. Blume, How noise matters, Games Econ. Behav. 44 (2003), 251-271. 
[3] J. Bricmont and J. Slawny, First order phase transitions and perturbation theory, in: Statistical Mechanics and Field Theory: Mathematical Aspects, Lecture Notes in Physics 257, Springer-Verlag, 1986.

[4] J. Bricmont and J. Slawny, Phase transitions in systems with a finite number of dominant ground states, J. Stat. Phys. 54 (1989), 89-161.

[5] R. Cressman, Evolutionary Dynamics and Extensive Form Games, MIT Press, Cambridge, USA, 2003.

[6] G. Ellison, Learning, local interaction, and coordination, Econometrica 61 (1993), 10471071.

[7] G. Ellison, Basins of attraction, long-run stochastic stability, and the speed of step-by-step evolution, Rev. Econ. Stud. 67 (2000), 17-45.

[8] D. Foster and P. H. Young, Stochastic evolutionary game dynamic, Theor. Pop. Biol. 38 (1990), 219-232.

[9] M. I. Freidlin and A. D. Wentzell, Random Perturbations of Dynamical Systems, Springer Verlag, New York, 1984.

[10] H. O. Georgii, Gibbs Measures and Phase Transitions, Walter de Gruyter, Berlin, 1988.

[11] C. Hauert and M. Doebeli, Spatial structure often inhibits the evolution of cooperation in the snowdrift game, Nature 428, (2004), 643-646.

[12] J. Hofbauer and K. Sigmund, Evolutionary Games and Population Dynamics, Cambridge University Press, Cambridge, 1998.

[13] J. Hofbauer and K. Sigmund, Evolutionary game dynamics, Bull. Am. Math. Soc. 40 (2003), 479-519.

[14] M. Kandori, G. J. Mailath, and R. Rob, Learning, mutation, and long-run equilibria in games, Econometrica 61 (1993), 29-56.

[15] K. Lindgren and M. G. Nordahl, Evolutionary dynamics of spatial games, Physica D 75 (1994), 292-309.

[16] J. Maynard Smith, Evolution and the Theory of Games, Cambridge University Press, Cambridge, 1982.

[17] J. Miękisz, Statistical mechanics of spatial evolutionary games, Phys. A: Math. Gen. 37 (2004), 9891-9906.

[18] J. Miękisz Stochastic stability in spatial games, J. Stat. Phys. 117 (2004), 99-110.

[19] J. Miękisz, Stochastic stability in spatial three-player games, Physica A 343 (2004), 175184.

[20] J. Miękisz, Equilibrium selection in evolutionary games with random matching of players, J. Theor. Biol. 232 (2005), 47-53.

[21] M. A. Nowak and R. M. May, Evolutionary games and spatial chaos, Nature 359(1992), 826-829.

[22] M. A. Nowak and R. M. May, The spatial dilemmas of evolution, Int. J. Bifurcation Chaos 3 (1993), 35-78.

[23] M. A. Nowak, S. Bonhoeffer, and R. M. May, More spatial games, Int. J. Bifurcation Chaos 4 (1994), 33-56.

[24] M. A. Nowak and K. Sigmund, Evolutionary dynamics of biological games, Science 303 (2004), 793-799.

[25] M. A. Nowak, Evolutionary Dynamics, Harvard University Press, Cambridge, USA, 2006.

[26] A. Robson and F. Vega-Redondo, Efficient equilibrium selection in evolutionary games with random matching, J. Econ. Theory 70 (1996), 65-92. 
[27] L. Samuelson, Evolutionary Games and Equilibrium Selection, MIT Press, Cambridge, USA, 1997.

[28] W. H. Sandholm, Simple formulas for stationary distributions and stochastically stable states, Games Econ. Behav. 59 (2007), 154-162.

[29] Y. G. Sinai, Theory of Phase Transitions: Rigorous Results, Pergamon, Oxford, 1982.

[30] G. Szabó, J. Vukov, and A. Szolnoki, Phase diagrams for an evolutionary prisoner's dilemma game on two-dimensional lattices, Phys. Rev. E 72 (2005), 047107.

[31] G. Szabó and G. Fáth, Evolutionary games on graphs, Physics Reports 446 (2007), 97-216.

[32] F. Vega-Redondo, Evolution, Games, and Economic Behaviour, Oxford University Press, Oxford, 1996.

[33] J. Weibull, Evolutionary Game Theory, MIT Press, Cambridge, USA, 1995.

[34] H. P. Young, The evolution of conventions, Econometrica 61 (1993), 57-84.

[35] H. P. Young, Individual Strategy and Social Structure: An Evolutionary Theory of Institutions, Princeton University Press, Princeton, 1998. 